\title{
COMPORTAMENTO DE LACTENTES NASCIDOS A TERMO PEQUENOS PARA A IDADE GESTACIONAL NO PRIMEIRO TRIMESTRE DE VIDA
}

\author{
Bernadete Balanin A. M ello르, Vanda M. Gimenes Gonçalves², Elisabete Abib P. Souza ${ }^{3}$
}

\begin{abstract}
RESUMO - O objetivo foi comparar o comportamento de lactentes nascidos a termo com peso adequado (AIG) a lactantes pequenos para a idade gestacional (PIG), no primeiro trimestre de vida. A amostra foi de 20 lactentes, avaliadosno 1ㅇ, 20 e 3을. meses. Foram utilizadas as Escalas Bayley de Desenvolvimento Infantil - II, com ênfase na Escala de Classificação do Comportamento (ECC). Houve diferença significativa entre os grupos no 2 o mês, com maior número de lactentes PIG classificados como alterados na ECC. O Fator Qualidade Motora demonstrou valores da mediana significativamente menores no grupo PIG, nos itens Motricidade Axial, Controle de Movimentos e Hipertonia Muscular. O Fator AtençãoNigília não mostrou diferença entre os grupos. Entretanto, quando analisados os itens Exploração de Objetos e de Ambiente e Interação com o Examinador, houve diferença significativa no 2o mês, com valores da mediana menores no grupo PIG.
\end{abstract}

PALAVRAS-CHAVE: retardo do crescimento intra-uterino, desnutrição intra-utero, comportamento de lactentes, neurodesenvolvimento.

\begin{abstract}
Behavior of full term infants small for gestational age in the first three months of life
ABSTRACT - The objective was to compare the behavior of full-term infants small-for-gestational age (SGA) with full-term appropriate-for-gestational age (AGA). The sample considered 20 infants in the $1^{\text {st }}, 2^{\text {nd }}$ and in the $3^{\text {rd }}$ months of life. The Bayley Scales of Infant Development-II were used, with attention to items related to Behavior Rate Scale (BRS). It was found that SGA infants showed lower average values in the BRSin the $2^{\text {nd }}$ month. The M otor Quality Factor displayed significantly lower average values in SGA group, in the items Gross-motor Movement Required by Tasks, Control of Movements and Hypertonicity. The Attention/Arousal Factor in the items Exploration of Objects/Surroundings and Orientation to Examiner displayed significantly lower average values in the SGA group.
\end{abstract}

KEY WORDS: intrauterine growth retardation, intrauterine malnutrition, infants behavior, neurodevelopment.

De acordo coma Academia Americana de Pediatria e o Colégio Americano de Obstetrícia e Ginecologia, todo neonato deve ser classificado segundo a idade intra-uterina e o peso, para efeito do relatório-padrão das estatísticas de saúde da reprodução e como pré-requisito para a determinação da normalidade ${ }^{1}$. No Brasil, o procedimento adotado na maioria das maternidadesé o mesmo, sendo rotina o diagnóstico da adequação peso/idade gestacional ${ }^{2}$. $\mathrm{Na}$ década de 60, estudos contribuíram para a dassificação da curva de crescimento fetal do recémnascido a termo ${ }^{3,4}$, que foi ampla e universalmente utilizada como referência por diferentes pesquisa- dores, induindo osbrasileiros. Foi determinado como limite inferior de peso adequado da curva de crescimento fetal o percentil 10, abaixo do qual os neonatosforam dassificados como pequenospara a idade gestacional (PIG). Entretanto, o conceito do neonato PIG é de definição estatística. Em estudos recentes, o termo PIG foi utilizado para identificar fetosque falharam em atingir um padrão de peso ou antropométrico arbitrário para determinada idade gestacional, com a ressalva de que alguns neonatos PIG poderiam ser constitucionalmente pequenos, representando a porção final da curva de distribuição normal do crescimento intra-uterino ${ }^{5}$.

\footnotetext{
Centro de Estudos e Pesquisas em Reabilitação "Prof. Dr. Gabriel Porto" (CEPRE); Departamento de Neurologia; Centro de Investigação em Pediatria (CIPED) Faculdade de Ciências Médicas (FCM)/Universidade Estadual de Campinas (UNICAMP) Campinas SP, Brasil: ${ }^{1}$ Psicóloga, Doutoranda em Ciências Médicas, CEPRE/FCM ; ${ }^{2}$ Neurologista Infantil, Professora Livre Docente do Departamento

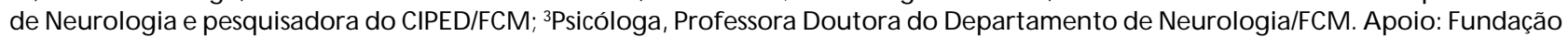
de Amparo Pesquisa do Estado de São Paulo (FAPESP) e Fundo de Apoio à Pesquisa (FAEP/Unicamp)
}

Recebido 28 Abril 2003, recebido na forma final 3 Maio 2004. Aceito 2 Julho 2004.

Dra. Bernadete B. Almeida Mello - Rua Adolfo Lutz 126 - 13084-880 Campinas SP - Brasil. E-mail: berna@unicamp.br 
O desenvolvimento de recém-nascidos PIG tem sido alvo constante de pesquisas, por ser considerado modelo de desnutrição em idade mais precoce. A partir das décadas de 60 e 70, pesquisadores tiveram sua atenção voltada para a percepção de que metade das crianças do mundo estava sofrendo de algum grau de desnutrição e que tal condição poderia limitar permanentemente sua capacidade intelectual num mundo avançado tecnologicamente ${ }^{6}$. Esta preocupação estava justificada frente aos experimentosem animais, que demonstravam a existência de um período crítico no desenvolvimento do sistema nervoso central (SNC) em que a desnutrição poderia causar danos irreversíveis, não só reduzindo o crescimento do cérebro, bem como deixando-o permanentemente menor em tamanho 7,8 . Períodos críticosno desenvolvimento do SNC são apontados como fasestemporais em que eventos bem definidos, originados extrinsecamente ao organismo, podem causar impacto permanentemente na organização e na função dos circuitos cerebrais $^{9}$. A chave desse conceito é a noção de que alterações idênticas que ocorram mais precoce ou mais tardiamente falham em produzir essas alterações irreversíveis bem definidas.

As vulnerabilidades potenciais do desenvolvimento do cérebro da criança são amplamente reconhecidas; no entanto, relativamente pouco se conhece a respeito dos mecanismos envolvidos. Osresultados de experimentos em animais podem ser estendidos apenas cautelosamente aos seres humanos, mas alguns desses achados certamente têm implicações clínicas importantes. As teorias contemporâneas enfatizam o potencial de autoorganização das estruturas cerebrais, particularmente das regiões envolvidas no armazenamento de informações, qual seja, a plasticidade em resposta a experiência ${ }^{10}$. Há vasta literatura mostrando a associação entre a inadequação peso ao nascimento/idade gestacional e o maior risco de morbidade neurológica, incluindo desde danos cerebrais permanentes, como a paralisia cerebral e o retardo mental ${ }^{11}$, até formas sutis de atrasos de desenvolvimento ${ }^{12}$. Entretanto, a maioria dos estudos em nascidos PIG foi realizada nas idades escolares, adolescentese adultos ${ }^{12,13}$ sendo observadas disfunções neurológicasmínimas, performance intelectual e psicológica pobres dificuldadesescolares, hiperatividade e incoordenação motora. Entre estudos realizadosno Brasil em neonatosou lactentes nascidos PIG, foi demonstrado exame neurológico neonatal alterado ${ }^{14,15}$, ou neurodesenvolvimento alterado nos $6^{\circ}, 12^{\circ}$ e $24^{\circ}$ meses de vida ${ }^{16-20}$. No re- ferente à organização dos estados comportamentais nos primeiros meses dos lactentes PIG, foram de especial interesse por providenciarem um índice da organização de múltiplas medidasneurológicas. Os índices de organização dos estados comportamentais no período neonatal foram relacionados com a disfunção do desenvolvimento posterior ${ }^{21,22}$. No período neonatal de nascidosPIG ${ }^{23-25}$, foi observada diferença significativa no processo interativo e no comportamento motor, demonstrando baixos níveis de atividade, poucas respostas ao estímulo, não interagindo tanto com examinador ou objetos inanimadose quando manipulados demonstraram estresse, desconforto e exaustão. Foi observada também hipotonia muscular.

Considerando as importantes diferenças referidas, o objetivo deste estudo foi a comparação do comportamento de lactentes nascidos PIG e aqueles adequados para a idade gestacional (AIG), frente a estímulos padronizados, acompanhados no primeiro trimestre de vida.

\section{MÉTODO}

Este é estudo analítico, prospectivo, numa população de lactentes nascidos a termo, com peso de nascimento PIG ou AIG, selecionados no Setor de Neonatologia do Centro de Atenção Integral à Saúde da Mulher (CAISM), da Universidade Estadual de Campinas (UNICAMP), no período de Setembro de 2000 a Agosto de 2001 . O projeto de pesquisa foi aprovado pelo Comitê de Ética em Pesquisa da UNICAMP e os responsáveis pelosneonatosassinaram o Termo de Consentimento Livre e Esclarecido.

Foram incluídosneonatos com idade gestacional entre 37 e 41 semanas, mantidosem alojamento conjunto. A adequação peso/idade gestacional ${ }^{3}$ dassificou no grupo AIG o recém-nascido com peso de nascimento entre ospercentis 25 e 90 do valor de referência e como grupo PIG aquele com peso de nascimento abaixo do percentil 10. Foram excluídas as malformações congênitas ou síndromes genéticas diagnosticadas no berçário e asinternações em unidade de terapia intensiva neonatal.

Retornou para avaliações mensais a população de 46 lactentes. Para este estudo foi considerada a amostra de coorte longitudinal de 20 lactentes que compareceram aos retornos mensais durante o primeiro trimestre de vida, sem nenhuma falta. O grupo AIG foi constituído por 11 neonatos e o grupo PIG por 9 neonatos.

Como teste padronizado, utilizou-se as Bayley Scales of Infant Development II (BSID-II) ${ }^{26}$, constituída de três escalas: Mental, Motora e de Classificação do Comportamento. A dassificação doslactentesnasBSID-II baseouse no Index Score (IS) dasEscalas Mental e Motora e Raw Score (RS) da Escala de Classificação do Comportamento (ECC), de acordo com a performance neuropsicomotora da criança, em cada mês de avaliação. 
Um sistema de terminologia bem definido no manual dasescalas considerou a pontuação dasBSID-II com a média de 100 e o desvio-padrão de 15. Foi definida a classificação do IScomo: dentro doslimites normais (ISentre 85 e 114); performance levemente alterada (IS entre 70 e 84) e performance significativamente alterada (IS $\leq 69$ ) e performance acelerada (IS $\geq 115$ ).

A Escala de Classificação do Comportamento (ECC), observou de forma direta o comportamento do lactente frente aos estímulos padronizados recomendados, complementando e facilitando a interpretação das Escalas Mental e Motora, durante a aplicação da BSID-II. A ECC avaliou no primeiro trimestre de vida, doisfatores: Atenção/Vigília e Qualidade Motora.

O Fator Atenção/Vigília consistiu de 9 itens: o estado predominante, a labilidade do estado de alerta/sonolência, afeto positivo, afeto negativo, o acalmar-se, energia, interesse na avaliação, exploração dosobjetose orientação para o examinador. O Fator Qualidade Motora consistiu de 7 itens: motricidade axial, coordenação apendicular, controle de movimentos, hipotonia e hipertonia muscular, tremor, movimentos lentos e frenéticos. As respostas em cada item, corresponderam a um valor que variou de 1 a 5 , que foram somados.

O sistema de terminologia definido no manual das escalas transformou o número de créditos na ECC em RS, que apósfoi transformado em Percentil, com a seguinte classificação: RS dentro dos limites normais $\geq 26$; RS questionável entre 25 e 10 e RS não-ótimo $\leq 11$. Considerou-se como normal as pontuações de RS $\geq 26$ e alterada as pontuações $<26$.

Para descrever osgruposforam apresentadastabelas de freqüência das variáveis categóricas e medidas de posição e dispersão das variáveiscontínuas. Para comparar os grupos através das variáveis contínuas foi utilizado o teste de Mann-Whitney e para comparar os grupos

Tabela 1. Escala Mental nosgruposAIG e PIG no primeiro trimestre de vida.

\begin{tabular}{lll}
\hline Meses $\quad$ Mediana & Desvio Padrão \\
\hline
\end{tabular}

Escala Mental $\mathrm{n}=11$

Grupo AIG

$\begin{array}{llc}\text { 1o mês } & 87 & 13,04 \\ \text { 2o mês } & 94 & 9,38 \\ \text { 3o mês } & 89 & 11,77\end{array}$

Escala Mental $n=9$

Grupo PIG

\begin{tabular}{llcl} 
1o mês & 92 & 9,17 & \\
2o mês & 84 & 11,00 & $0,048^{*}$ \\
3o mês & 87 & 8,03 & \\
\hline
\end{tabular}

AIG, adequado para a idade gestacional; PIG, pequeno para a idade gestacional; *, diferença significativa; ${ }^{\mathrm{b} T e s t e ~ d e ~ F r i e d m a n . ~}$ através das variáveis categóricas foi utilizado o teste exato de Fisher. Para comparação múltipla foi utilizado o teste de Friedman. O nível de significância foi $5 \%$.

\section{RESULTADOS}

A amostra do estudo longitudinal foi composta por 20 lactentes, sendo 11 do grupo AIG e 9 do grupo PIG. Comparando os valores da mediana intragrupo do IS na Escala Mental, houve diferença estatisticamente significativa ( $p=0,048$ ) no grupo PIG, com a maior pontuação observada no 10 mês e a menor pontuação no $2^{\circ}$ mês de vida, conforme a Tabela 1.

Comparando osvalores da mediana intra-grupo do IS na Escala Motora, houve diferença significativa nos dois grupos, entre o 20 e o 30 meses de vida (no grupo AIG, $p=0,003$ e no grupo PIG, $p=0,008$ ). No grupo PIG os valores da mediana foram mais elevados no $1^{\text {o }}$ mês, diminuindo progressivamente até o 3o mês, conforme a Tabela 2.

A análise da comparação dos grupos quanto à classificação normal ou alterada na ECC encontrase na Tabela 3, que mostrou diferença significativa entre os grupos no $2^{\circ}$ mês $(p=0,026)$. No 1 o mês de vida, observou-se que, embora a diferença não fosse significativa, os lactentes PIG apresentaram maior freqüência de dassificação normal, podendo sugerir melhores resultadosque osAIG. No 3o mês, os resultados foram iguais entre os grupos.

$\mathrm{Na}$ avaliação qualitativa, no primeiro trimestre de vida observou-se alteração no comportamento dos lactentes durante a aplicação da escala, taiscomo: manifestação de choro e irritabilidade quando o lactente estava com fome, sono ou com algum

Tabela 2. Escala Motora, nos grupos AIG e PIG no primeiro trimestre de vida.

\begin{tabular}{lll}
\hline Meses $\quad$ Mediana & Desvio Padrão & -valor \\
\hline
\end{tabular}

Escala Motora $n=11$

Grupo AIG

$\begin{array}{lcc}\text { 1o mês } & 97 & 7,98 \\ \text { 2o mês } & 99 & 6,57 \\ \text { 3o mês } & 91 & 10,11 \\ \text { Escala Motora } & \mathrm{n}=9 & \end{array}$

$0,003^{*}$

Escala Motora $\mathrm{n}=9$

\begin{tabular}{llll} 
1o mês & 97 & 8,67 & \\
2o mês & 87 & 8,35 & $0,008^{*}$ \\
3o mês & 82 & 8,09 & \\
\hline
\end{tabular}

AIG, adequado para a idade gestacional; PIG, pequeno para a idade gestacional; *, diferença significativa; ${ }^{\mathrm{b}}$ Teste Friedman. 
Tabela 3. Distribuição da dassificação da ECC, segundo os gruposno primeiro trimestre de vida.

\begin{tabular}{lcccc}
\hline Meses & Grupos & Normal & Alterado & p-valora \\
\hline 1o mês & AIG $(n=11)$ & 7 & 4 & 0,642 \\
& PIG $(n=9)$ & 7 & 2 & \\
2o mês & AIG $(n=11)$ & 11 & 0 & $0,026^{*}$ \\
& PIG $(n=9)$ & 5 & 4 & \\
3o mês & AIG $(n=11)$ & 10 & 1 & 1,000 \\
& PIG $(n=9)$ & 8 & 1 & \\
\hline AIG
\end{tabular}

AIG, adequado para a idade gestacional; PIG, pequeno para a idade gestacional; ECC escala de classificação do Comportamento; * , diferença significativa; ateste de Fisher.

desconforto físico ou falta de interesse nosmateriais do teste.

Asmanifestações desses comportamentosforam observadas nos lactentes de ambos os grupos e, quando mais intensas, levaram à interrupção da avaliação do neurodesenvolvimento. Quando menosintensase passíveis de serem acalmadas, não impediram que os lactentes dos dois grupos demonstrassem suas capacidades de interação, estabilidade e equilíbrio frente aos estímulos do meio.

Analisando osfatores da ECC que diferenciaram os grupos no $2^{\circ}$ mês, verificou-se que algunsitens mostraram diferença significativa. Conforme a Tabela 4, na análise dos valores da mediana do RS do Fator Atenção/Nigília, no $2^{\circ}$ mês de vida, ositens exploração de objetos/ambiente e interação com o examinador, mostraram valores medianos menores no grupo PIG.

A análise dos valores da mediana do RS do Fator Qualidade Motora, no $2^{\circ}$ mês de vida, mostrou diferença significativa nositens motricidade axial, controle de movimentos e hipertonia muscular, com valores medianos menores no grupo PIG.

\section{DISCUSSÃO}

Foi referida a grande dificuldade diagnóstica no lactente, em função da questão do desenvolvimento, que propicia mudança contínua nas características do sujeito ${ }^{27}$, sendo necessário o uso de testese escalas enquanto medidasobjetivas e padronizadas de comportamento. Novos instrumentos têm sido elaborados para a avaliação psicopatológica da criança que se encontra no período sensório motor. Recentemente, foi validado em lactentes brasileirosnormais um instrumento de rastreamento de possíveis casos psiquiátricos ${ }^{27}$, que avaliou comportamentos espontâneos, após a reação aos estímulose a evolução das reaçõesno decorrer do exame. Os autores enfatizaram a importância do

Tabela 4. Fator Atenção/Nigília e Qualidade Motora no $2^{\circ}$ mês de vida.

\begin{tabular}{|c|c|c|c|c|c|}
\hline Item & Grupo & média & DP & Mediana & $\mathrm{p}$-valor ${ }^{\mathrm{a}}$ \\
\hline \multicolumn{6}{|c|}{ Fator Atenção/Vigília } \\
\hline \multirow[t]{2}{*}{ Exploração de objetos } & AIG $(n=11)$ & 4,64 & 0,67 & 5 & $0,036 *$ \\
\hline & PIG (n=9) & 3,50 & 1,41 & 4 & \\
\hline \multirow[t]{2}{*}{ Interação com o examinador } & AIG $(n=11)$ & 4,82 & 0,4 & 5 & $0,030^{*}$ \\
\hline & PIG $(n=9)$ & 4,00 & 0,93 & 4 & \\
\hline \multicolumn{6}{|c|}{ Fator Qualidade Motora } \\
\hline \multirow[t]{2}{*}{ Motricidade axial } & AIG $(n=11)$ & 4,82 & 0,4 & 5 & $0,001^{*}$ \\
\hline & PIG (n=9) & 3,22 & 1,48 & 3 & \\
\hline \multirow[t]{2}{*}{ Controle de movimentos } & AIG $(n=11)$ & 4,64 & 0,5 & 5 & $0,002^{*}$ \\
\hline & PIG (n=9) & 3,00 & 1,41 & 3 & \\
\hline Hipertonia muscular & AIG $(n=11)$ & 4,45 & 0,52 & 4 & $0,014^{*}$ \\
\hline
\end{tabular}


diagnóstico no período sensório motor, utilizando estimadores que considerem principalmente esquemas motorese de sociabilidade em sua apresentação mais primitiva. Esse trabalho tem sido aplicado a outras populações, garantindo a fidedignidade do instrumento.

Utilizando um instrumento padronizado internacional, não validado para o lactente brasileiro, observou-se na pontuação das escalas mental e motora no 1 o mês de vida, valores da mediana mais elevadosno grupo PIG, embora a diferença não fos se significativa. Entretanto, no 20 mês de vida, os resultados do IS mental e motor foram significativamente inferiores no grupo PIG. Assim, verificouse, com o uso deste instrumento de avaliação, a pos sibilidade de identificação do grupo PIG no 2 o mês de vida. Esses resultados poderiam indicar a assim chamada transformação maior da função neural, referida por volta do final do $2^{\circ}$ mês de vida na criança nascida de termo ${ }^{28}$. Nesse período, muitas funções neurais mudariam para uma condição mais adaptativa que durante os meses anteriores ${ }^{29}$.

Entendeu-se que a transformação da função neural levou no 20 mês à melhor performance do grupo AIG e que não tivesse ainda ocorrido no grupo PIG. Entretanto, no 3o mês de vida os doisgruposforam semelhantes. Esta preocupação sugeriu a existência de um período crítico no 2 $\%$ mês de vida, em que a desnutrição poderia causar impacto na organização e na função dos circuitos cerebrais.

Raramente têm sido referidos estudos sobre o neurodesenvolvimento de lactentes que apontassem diferenças no $2^{\circ}$ mês de vida. Em pesquisa semelhante foram avaliados indicadores de risco para lesão neurológica ${ }^{30}$ e verificou-se sua repercussão no desenvolvimento neuromotor no primeiro trimestre de vida, por meio dasBSID-II. Foi observada diferença significativa no $2 \circ$ mês entre os grupos expostosou não aosindicadores de risco. Do mesmo modo, foram acompanhados lactentes PIG e AIG no 1 o ano de vida, utilizando um instrumento composto de provas baseadas em avaliações neurológicase fisioterápicas provenientes de diferentes autores ${ }^{31}$. Foi observado no 20 mêsque os lactentes PIG apresentaram pontuação inferior aos AIG.

No que se refere aos estados comportamentais avaliados neste estudo, na análise da ECC não foi observada diferença entre os grupos no 1 으 e 3 은 meses. Entretanto no $2 \circ$ mês houve número significativamente maior de lactentes PIG classificados como alteradose alguns aspectos comportamentais influenciaram nesta classificação.
Analisando o Fator Atenção/Nigília, no $2^{\circ}$ mês de vida, os itens que mostraram diferença entre os gruposforam a exploração de objetos/ambiente e interação com o examinador, com valores medianos significativamente menores no grupo PIG. Achadossemelhantesforam referidosem lactentes PIG, que não interagiram facilmente com estímulos sociais, não focalizando nem modulando de maneira adequada a interação com o ambiente ${ }^{24}$. Foi observado que embora os lactentes entrassem em estado de alerta, a resposta a estímulos do meio foi pobre.

A respeito de aspectos comportamentais relacionadosà interação entre mãese lactentes PIG nos primeirosmeses de vida, foi demonstrado no $2 \stackrel{\circ}{\circ}$ mês maior dificuldade na labilidade de seu estado e altos níveis de sonolência comparados com os AIG. No 3o mês, os lactentes PIG levaram mais tempo para sair do estado de sonolência para o alerta22.

Além do Fator Atenção/Nigília, outro Fator considerado na ECC foi a Qualidade Motora, que mostrou diferença significativa entre osgruposno 20 mês, nositens motricidade axial, controle de movimentos e hipertonia muscular de membros, com valores da mediana inferiores nos lactentes PIG. Resultados semelhantes foram observados em PIG no período neonatal ${ }^{14,15}$, sendo referida a hiperexcitabilidade, associada a hipotonia axial ou hipertonia de membros. Esses achados foram referidos também no 3 으 mês de vida, quando foram observadas alterações neurológicasem $59,4 \%$ doslactentes, sendo a mais freqüente a hiperexcitabilidade, associada a hipotonia ou hipertonia muscular ${ }^{20}$.

Concluindo, as diferenças encontradas entre os dois grupos no 1 o trimestre de vida sugeriram a possibilidade de identificar alteraçõesem idades precoces, que parecem ser transitórias. Esses achadosindicaram a necessidade de estudos por maior período de acompanhamento, para entender sua repercussão tardia.

\section{REFERÊNCIAS}

1. Fletcher MA. Avaliação física e classificação. In Fletcher MA. Neonatologia: fisiopatologia e tratamento do recém-nascido. 4.Ed. São Paulo: Medsi, 1999.

2. Goto MMF, Gonçalves VMG, Aranha A Netto. Classificação do recémnascido e implicações clínicas: aspectos relacionados ao peso ao nascimento. Temas sobre Desenvolvimento 2004;13.

3. Lubchenco LO, Hansman,C, Dressler M, Boyd E. Intrauterine growth as estimated from liveborn birth-weight data at 24 to 42 weeks of gestation. Pediatrics 1963;11:793-800.

4. Battaglia FC, Lubchenco LO. A practical classification of newborn infants by weight and gestational age. J Pediat 1967;71:159-163.

5. Goldenberg RL, Cliver SP. Small for gestational age and intrauterine growth restriction: definitions and standards. Clin Obstet Gynecol 1997;40:704-714. 
6. Levitsky DA, Strupp BJ. Malnutrition and the brain: changing concepts, changing concerns. J Nutr 1995;125(Suppl):2212-2220.

7. Dobbing J, Sands J. Vulnerability of developing brain: the effect of nutritional growth retardation on the timing of the brain growth-spurt. Biol Neonate 1971;19:363-378.

8. Winick M. Nutrition, growth and mental development: biological correlation. Am J Dis Child 1970;120:416-418.

9. Goodman CS, Shatz CJ. Developmental mechanisms that generate precise patterns of neuronal connectivity. Cell 72/Neuron 1993;10(Suppl):77-98.

10. Black JE. How a child builds its brain: some lessons from animal studies of neural plasticity. Prev Med 1998;27:168-171.

11. Nelson KB, Broman SH. Perinatal risk factors in children with serious motor and mental handcaps. Ann Neurol 1977;2:371-377.

12. Strauss RS. Adult functional outcome of those born small for gestational age: twenty-six-year follow-up of the 1970 British birth cohort. JAMA 2000;283:625-632.

13. Guardiola A, Egewarth C, Rotta NT. Avaliação do desenvolvimento neuropsicomotor em escolares de primeira série e sua relação com o estado nutricional. Jornal de Pediatria (Rio Janeiro) 2001;77:189-196.

14. Gherpelli, JLD. Evolução neurológica do recém-nascido pequeno para a idade gestacional: estudo dos fatores de risco relacionados com o prognóstico neurológico durante o primeiro ano de vida. Tese (Doutorado) Universidade de São Paulo São Paulo, 1988.

15. Oliveira KF. O exame neurológico de recém-nascidos pequenos para a idade gestacional, comparado ao de recém-nascidos adequados. Neurobiologia 1997;60:75-90.

16. Eickmann SH, Lima MC. Desenvolvimento mental e motor aos 24 meses de crianças nascidas a termo com baixo peso. Arq Neuropsiquiatr 2002;60:748-754.

17. Gagliardo HGRG. Avaliação das funções visuomotoras em lactentes a termo pequenos para a idade gestacional no primeiro semestre de vida. Tese (Doutorado) Universidade Estadual de Campinas Campinas, 2003.
18. Grantham-McGregor SM, Lira PI, Morris SS, Assunção AM. The development of low birth weight term infants and effects of the environment in Northeast Brazil. J Pediatr 1998;132:661-666.

19. Morris S, Grantham-McGregor SM, Lira PC, Assunção AM, Ashworth A. Effect of breastfeeding and morbidity on the development of low birthweight term babies in Brazil. Acta Paediatr 1999;88:1101-1106.

20. Gherpelli JLD, Ferreira H, Costa PFH. Neurological follow-up of smallfor-gestational age newborn infants. Arq Neuropsiquiatr 1993;51:50-58.

21. Stern E, Parmelee AN, Akiyama Y, Schultz MZ, Wenner WH. Sleep cycle characteristics in infants. Pediatrics 1969;43:65-70.

22. Watt J, Strongman KT. Mother-infant interaction at 2 and 3 months in preterm, small-for-gestational-age, and full-term infants; their relationship with cognitive development at 4 months. Early Hum Dev 1985;11:231-246.

23. Michaelis R, Schulte FJ, Nolte R. Motor behavior of small for gestational age newborn infants. Pediatrics 1970;76:208-213.

24. Als H, Tronick E, Adamson L, Brazelton TB. The behavior of the full-term but underweight newborn infant. Dev Med Child Neurol 1976;18:590-602.

25. Abrol P, Kappor R, Gathwala G, Tiwari AD. Neonatal behavior in full term small for date. Indian Pediat 1994;31:785-789.

26. Bayley N. Bayley scales of infant development. 2.Ed. San Antonio: Harcourt, 1993.

27. Assumpção FB Jr, Kuczynski E, Rego MGS, Rocca CCA. Escala de avaliação da reação de retração no bebê: um estudo de validade. Arq Neuropsiquiatr 2002;60:56-60.

28. Prechtl HFR. Continuity of neural functions from prenatal to postnatal life. Clin Dev Med 1984;94.

29. Prechtl HFR. New perspectives in early human development. Eur J Obstet Gynecol Reprod Biol 1986;21:347-355.

30. Ravanini SG. Avaliação neuromotora de lactentes e indicadores de risco para lesão neurológica: análise qualitativa. Dissertação (Mestrado) Universidade Estadual de Campinas, Campinas, 1998.

31. Ounsted M, Moar VA, Scott A. Neurological development of small-forgestational age babies during the first year of life. Early Hum Dev 1998;16:163-172. 